\title{
Oriented by Characteristic Roots Reduced Matrices in the Class of Semiscalarly Equivalent
}

\author{
B. Z. Shavarovskii \\ Department of Aldebra, Pidstryhach Institute for Applied Problems of Mechanics and Mathematics, \\ National Academy of Sciences of Ukraine, Lviv 79060, Ukraine
}

Correspondence should be addressed to B. Z. Shavarovskii; bshavarovskii@gmail.com

Received 15 February 2021; Revised 23 March 2021; Accepted 5 April 2021; Published 19 April 2021

Academic Editor: Shaofang Hong

Copyright $\odot 2021$ B. Z. Shavarovskii. This is an open access article distributed under the Creative Commons Attribution License, which permits unrestricted use, distribution, and reproduction in any medium, provided the original work is properly cited.

A set of polynomial $3 \times 3$-matrices of simple structure has been singled out, for which a so-called oriented by certain characteristic roots reduced matrix is established in the class of semiscalarly equivalent. The invariants of such reduced matrices and the conditions of their semiscalar equivalence are indicated. The obtained results will also be applied to the problem of similarity of sets of numerical matrices.

\section{Introduction}

By Theorem 1 (see Section 4 in [1] and [2]), matrix $F(x) \in M(n, \mathbf{C}[x])$ of full rank by transformation

$$
F(x) \longrightarrow P F(x) Q(x)=G(x),
$$

where $P \in G L(n, C)$ and $Q(x) \in G L(n, C[x])$ are reduced to the lower triangular form with invariant factors on the main diagonal. We consider the case when $F(x)$ is a $3 \times 3$-matrix of simple structure and its first invariant factor is equal to one.

The latter condition does not limit the generality in the sense that everything stated in this article can be extended to $3 \times 3$-matrices, which after reduction (i.e., division of all their elements) by the first invariant factor become matrices of simple structure. By the definition of a matrix of simple structure, all its elementary divisors are linear. This concept is taken from [1], where it is introduced for polynomial matrices of arbitrary degree by analogy with the concept of numerical matrices (above the field) of simple structure with [3]. Note that the numerical matrices of a simple structure in [4] are called simple and in [5] are nondefective. Next, we will use the terminology from $[1,2]$, namely, the transformation (1) and the matrices $F(x)$ and $G(x)$ will be called semiscalarly equivalent (abbreviation: ssk.e., notation:
$F(x) \approx G(x))$. Our task is to construct in the class $\{P F(x) Q(x)\}$ a lower triangular matrix with invariant factors on the main diagonal and some predefined properties, to find a complete system of its invariants and to establish on this basis the conditions of ssk.e. for two such matrices. This problem for $2 \times 2$-matrices in the general case is solved by the author in [6] and for $3 \times 3$-matrices with one characteristic root in [7].

\section{Preliminary Information}

In class $\{P F(x) Q(x)\}$, there is a matrix of the form

$$
A(x)=\left\|\begin{array}{ccc}
1 & 0 & 0 \\
a_{1}(x) & \phi_{1}(x) & 0 \\
a_{3}(x) & a_{2}(x) & \phi_{2}(x)
\end{array}\right\|,
$$

where $\quad \operatorname{deg} a_{1}(x)<\operatorname{deg} \phi_{1}(x), \quad \operatorname{deg} a_{2}(x), \operatorname{deg} a_{3}(x)<$ $\operatorname{deg} \phi_{2}(x)$, and $\phi_{1}(x)$ divides $a_{2}(x)$ and $\phi_{2}(x)$. Let us denote $\phi_{12}(x):=\phi_{2}(x) / \phi_{1}(x)$ and $a_{2}^{\prime}(x):=a_{2}(x) / \phi_{1}(x)$. We assume that $\operatorname{deg} \phi_{1}(x), \operatorname{deg} \phi_{12}(x)>1$. Otherwise, $A(x)$ in class $\{P F(x) Q(x)\}$ can be chosen so that $a_{1}(x) \equiv 0$ or $a_{2}(x) \equiv 0$. In these cases, the task of finding the conditions ssk.e. matrices are greatly simplified. Hereinafter, the notation $M_{1}$ and $M_{2}$ will mean the sets of roots of polynomials $\phi_{1}(x)$ and $\phi_{12}(x)$, respectively. Recall that element 
$\alpha \in M_{1} \cup M_{2}$ is called the characteristic root of the matrix $A(x)$ and $M_{1} \cup M_{2}$ for class $\{P F(x) Q(x)\}$ is defined unambiguously (i.e., does not depend on the choice of matrix $A(x)$ ). Obviously, $A(x)$ in class $\{P F(x) Q(x)\}$ can be chosen so that, for some $\alpha_{0} \in M_{1}$, the following condition

$$
a_{1}\left(\alpha_{0}\right)=a_{3}\left(\alpha_{0}\right)=0
$$

is satisfied. In the future, this condition will be mandatory for $A(x)$ and for all triangular matrices of class $\{P F(x) Q(x)\}$. Hereinafter, record $(a(x), b(x))$ will mean the greatest common divisor of polynomials $a(x)$ and $b(x)$. We will use the corresponding notation for the greatest common divisor of three polynomials.

Proposition 1. The greatest common divisor $\left(a_{1}(x), a_{3}(x), \phi_{1}(x)\right)$ for class $\{P F(x) Q(x)\}$ does not depend on the choice of the matrix $A(x)$ with a fixed characteristic root $\alpha_{0} \in M_{1}$.

Proof. Let the matrix

$$
B(x)=\left\|\begin{array}{ccc}
1 & 0 & 0 \\
b_{1}(x) & \phi_{1}(x) & 0 \\
b_{3}(x) & b_{2}(x) & \phi_{2}(x)
\end{array}\right\|
$$

where $\quad \operatorname{deg} b_{1}(x)<\operatorname{deg} \phi_{1}(x), \quad \operatorname{deg} b_{2}(x), \operatorname{deg} b_{3}(x)<\operatorname{deg} \phi_{2}$ $(x)$, and $b_{1}\left(\alpha_{0}\right)=b_{3}\left(\alpha_{0}\right)=0$, belongs to class $\{P F(x) Q(x)\}$.

Then, for some matrices $\left\|s_{i j}\right\|_{1}^{3} \in G L(3, C)$ and $\left\|r_{i j}(x)\right\|_{1}^{3} \in G L(3, C[x])$, the equality

$$
\left\|s_{i j}\right\|_{1}^{3}\left\|\begin{array}{ccc}
1 & 0 & 0 \\
a_{1}(x) & \phi_{1}(x) & 0 \\
a_{3}(x) & a_{2}(x) & \phi_{2}(x)
\end{array}\right\|=\left\|\begin{array}{ccc}
1 & 0 & 0 \\
b_{1}(x) & \phi_{1}(x) & 0 \\
b_{3}(x) & b_{2}(x) & \phi_{2}(x)
\end{array}\right\|\left\|r_{i j}(x)\right\|_{1}^{3}
$$

holds. From the latter, we can write

$$
\begin{gathered}
r_{11}(x)=s_{11}+s_{12} a_{1}(x)+s_{13} a_{3}(x), \\
s_{21}+s_{22} a_{1}(x)+s_{23} a_{3}(x)=b_{1}(x) r_{11}(x)+\phi_{1}(x) r_{21}(x), \\
s_{31}+s_{32} a_{1}(x)+s_{33} a_{3}(x)= \\
b_{3}(x) r_{11}(x)+b_{2}(x) r_{21}(x) \\
+\phi_{2}(x) r_{31}(x) .
\end{gathered}
$$

If we put in (7) and (8) $x=\alpha_{0}$, we get $s_{21}=0$ and $s_{31}=0$, respectively. Since $\phi_{1}(x)$ divides $r_{12}(x)$ and $r_{13}(x)$, then $\left(r_{11}(x), \phi_{1}(x)\right)=1$. Therefore, from (7) and (8), we have that $\left(a_{1}(x), a_{3}(x), \phi_{1}(x)\right)$ divides $b_{1}(x)$ and $b_{3}(x)$. Given that ssk.e. has the property of symmetry (moreover, ssk.e. is the ratio of equivalence), we can write that $\left(b_{1}(x), b_{3}(x), \phi_{1}(x)\right)$ divides $a_{1}(x)$ and $a_{3}(x)$. Therefore, $\left(a_{1}(x), a_{3}(x), \phi_{1}(x)\right)=\left(b_{1}(x), b_{3}(x), \phi_{1}(x)\right)$. The proposition is proved.

In this study, we will limit ourselves to the case when $\left(a_{1}(x), a_{3}(x), \phi_{1}(x)\right)=\phi_{1}(x)$. Then, $a_{1}(x) \equiv 0$ and $\phi_{1}(x)$ divides $a_{3}(x)$. Considering the opposite case, when $\left(a_{1}(x), a_{3}(x), \phi_{1}(x)\right) \neq \phi_{1}(x)$, is the subject of another study.

\section{Invariants of Triangular Matrices}

Proposition 2. If $\left(a_{1}(x), a_{3}(x), \phi_{1}(x)\right)=\phi_{1}(x)$, then $\left(a_{3}(x), \phi_{2}(x)\right)$ for class $\{P F(x) Q(x)\}$ does not depend on the choice of matrix $A(x)$.

Proof. Let $B(x)$ (4) be some other matrix of class $\{P F(x) Q(x)\}$ for which a condition similar to (3) holds. Since $a_{1}(x)=b_{1}(x) \equiv 0$, then, from (6) and (7), we have $r_{11}(x)=s_{11}+s_{13} a_{3}(x)$ and $r_{21}(x)=s_{23} a_{3}(x) / \phi_{1}(x)$. Then, (8), where $s_{31}=0_{1}$, can be written as

$$
\begin{aligned}
s_{33} a_{3}(x)= & b_{3}(x)\left(s_{11}+s_{13} a_{3}(x)\right)+s_{23} b_{2}(x) \frac{a_{3}(x)}{\phi_{1}(x)} \\
& +\phi_{2}(x) r_{31}(x) .
\end{aligned}
$$

Since $s_{11} \neq 0$, the latter shows that $\left(a_{3}(x), \phi_{2}(x)\right)$ divides $b_{3}(x)$. Therefore, given the symmetry of the ratio nsk.e., we have that $\left(b_{3}(x), \phi_{2}(x)\right)$ divides $a_{3}(x)$ and finally $\left(a_{3}(x), \phi_{2}(x)\right)=\left(b_{3}(x), \phi_{2}(x)\right)$. The proposition is proved.

We divide the set $M_{2}$ of roots of polynomial $\phi_{12}(x)$ into subsets according to the following principle: we assign elements $\alpha_{1}, \alpha_{2} \in M_{2}$ to the same subset if condition $\left\|a_{3}\left(\alpha_{1}\right) a_{2}^{\prime}\left(\alpha_{1}\right)\right\|=\left\|a_{3}\left(\alpha_{2}\right) a_{2}^{\prime}\left(\alpha_{2}\right)\right\|$ holds for $A(x)$. Thus, we get some partition of the set $M_{2}$ :

$$
M_{2}=K_{1} \cup \ldots \cup K_{m} .
$$

Proposition 3. Partition (10) of the set $M_{2}$ for class $\{P F(x) Q(x)\}$ does not depend on the choice of matrix $A(x)$ if $\left(a_{1}(x), a_{3}(x), \phi_{1}(x)\right)=\phi_{1}(x)$.

Proof. Suppose that, in addition to $A(x)$, the matrix $B(x)$ (4) with a condition similar to (3) also belongs to class $\{P F(x) Q(x)\}$. Then, for some $\left\|s_{i j}\right\|_{1}^{3} \in G L(3, C)$ and $\left\|r_{i j}(x)\right\|_{1}^{3} \in G L(3, C[x])$, equation (5) holds, from which we have (9) and

$$
\begin{aligned}
s_{32}+s_{33} a_{2}^{\prime}(x)= & b_{3}(x)\left(s_{12}+s_{13} a_{2}^{\prime}(x)\right)+b_{2}^{\prime}(x) \\
& \cdot\left(s_{22}+s_{23} a_{2}^{\prime}(x)\right)+\phi_{12}(x) r_{32}(x),
\end{aligned}
$$

where $b_{2}^{\prime}(x):=b_{2}(x) / \phi_{1}(x)$. Let $\alpha_{1}$ and $\alpha_{2}$ belong to one of the subsets of (10), for example, to $K_{1}$. If $x=\alpha_{1}$ and $x=\alpha_{2}$ is put alternately and the obtained equations are subtracted in (9), then we get $\left(b_{3}\left(\alpha_{1}\right)-b_{3}\left(\alpha_{2}\right)\right)\left(s_{11}+s_{13} a_{3} \quad\left(\alpha_{1}\right)\right)+$ $\left(b_{2}^{\prime}\left(\alpha_{1}\right)-b_{2}^{\prime}\left(\alpha_{2}\right)\right) s_{23} a_{3}\left(\alpha_{1}\right)=0$.

Similarly, from (11), we obtain $\left(b_{3}\left(\alpha_{1}\right)-b_{3}\left(\alpha_{2}\right)\right)\left(s_{12}+\right.$ $\left.s_{13} a_{2}^{\prime}\left(\alpha_{1}\right)\right)+\left(b_{2}^{\prime}\left(\alpha_{1}\right)-b_{22}^{\prime}\left(\alpha_{2}\right)\right)\left(s_{22}+s_{23} a_{2}^{\prime}\left(\alpha_{1}\right)\right)=0$.

The last two equations are written in matrix form $\left\|b_{3}\left(\alpha_{1}\right)-b_{3}\left(\alpha_{2}\right) b_{2}^{\prime}\left(\alpha_{1}\right)-\quad b_{2}^{\prime}\left(\alpha_{2}\right)\right\| \| s_{11}+s_{13} a_{3}\left(\alpha_{1}\right) s_{12}+$ $s_{13} a_{2}^{\prime}\left(\alpha_{1}\right) s_{23} a_{3}\left(\alpha_{1}\right)_{22}+s_{23} a_{2}^{\prime}\left(\alpha_{1}\right) \|=0$.

It is easy to see that $\operatorname{det}\left\|\begin{array}{cc}s_{11}+s_{13} a_{3}\left(\alpha_{1}\right) & s_{12}+s_{13} a_{2}^{\prime}\left(\alpha_{1}\right) \\ s_{23} a_{3}\left(\alpha_{1}\right) & s_{22}+s_{23} a_{2}^{\prime}\left(\alpha_{1}\right)\end{array}\right\|=\operatorname{det}\left\|r_{i j}\left(\alpha_{1}\right)\right\|_{1}^{2}$. 
Since in $\left\|r_{i j}(x)\right\|_{1}^{3}$, for $\alpha_{1}$, we have $r_{13}\left(\alpha_{1}\right)=r_{23}\left(\alpha_{1}\right)=0$, then $\left\|r_{i j}\left(\alpha_{1}\right)\right\|_{1}^{2} \neq 0$. Therefore, $\left\|b_{3}\left(\alpha_{1}\right) b_{2}^{\prime}\left(\alpha_{1}\right)\right\|=$ $\left\|b_{3}\left(\alpha_{2}\right) \quad b_{2}^{\prime}\left(\alpha_{2}\right)\right\|$. The proposition is proved.

If, in (10), $m=1$, then the elements $a_{2}(x)$ and $a_{3}(x)$ in $A(x)$ are determined to the nearest multiplier, and then, $A(x)$ can be chosen so that $a_{2}(x) \equiv 0$. In this case, $A(x)$ can be considered canonical in class $\{P F(x) Q(x)\}$. Therefore, we consider the case when $m>1$.

\section{Reduction to a Special Triangular Form}

Proposition 4. Suppose that, for $A(x)$ (2), the conditions hold: $\left(a_{1}(x), a_{3}(x), \phi_{1}(x)\right)=\phi_{1}(x)$, in (10), $m>1$ and $a_{3}\left(\beta_{1}\right)$ or $a_{3}\left(\beta_{2}\right)$ are nonzero for $\beta_{1} \in K_{1}$ and $\beta_{2} \in K_{2}$. Then, in the class $\{P F(x) Q(x)\}$, there is a matrix $B(x)(4)$, in which $b_{3}\left(\beta_{1}\right) \neq b_{3}\left(\beta_{2}\right)$ and $b_{2}\left(\beta_{1}\right)=b_{2}\left(\beta_{2}\right)=0$.

Proof. We can assume that $a_{2}\left(\beta_{1}\right) \equiv 0$. Otherwise, this is achieved by adding the second row of matrix $A(x)$ multiplied by some number to its third row. If $a_{3}\left(\beta_{1}\right)=a_{3}\left(\beta_{2}\right) \neq 0$ and $a_{2}\left(\beta_{2}\right) \neq 0$, then, in the first step from $A(x)$, we move on to such a matrix $B(x)$ (4) in class $\{P F(x) Q(x)\}$ that $b_{3}\left(\beta_{1}\right) \neq b_{3}\left(\beta_{2}\right)$. To do this, choose $c_{1} \neq 0$ so that $1+c_{1} a_{2}^{\prime}\left(\beta_{i}\right) \neq 0$ for every $\beta_{i} \in M_{2}$. Then, from the congruence $a_{2}^{\prime}(x)-b_{2}^{\prime}(x)\left(1+c_{1} a_{2}^{\prime}(x)\right) \equiv 0\left(\bmod \phi_{12}(x)\right)$, we find $b_{2}^{\prime}(x), \operatorname{deg} b_{2}^{\prime}(x)<\operatorname{deg} \phi_{12}(x)$. Obviously, $b_{2}^{\prime}\left(\beta_{1}\right)=0$ and $b_{2}^{\prime}\left(\beta_{2}\right) \neq 0$. Next, from the congruence $a_{3}(x)-b_{3}(x)-c_{1} b_{2}^{\prime}(x) a_{3}(x) \equiv 0\left(\bmod \phi_{2}(x)\right)$, we find $b_{3}(x), \operatorname{deg} b_{3}(x)<\operatorname{deg} \phi_{2}(x)$. It is easy to see that $b_{3}\left(\beta_{1}\right)=$ $a_{3}\left(\beta_{1}\right)$ and $b_{3}\left(\beta_{2}\right) \neq a_{3}\left(\beta_{2}\right)$, since $c_{1} b_{2}^{\prime}\left(\beta_{2}\right) a_{3}\left(\beta_{2}\right) \neq 0$. Based on the above selected $c_{1}$ and obtained $b_{2}^{\prime}(x)$ and $b_{3}(x)$, we construct matrices $\left\|s_{i j}\right\|_{1}^{3}=\left\|\begin{array}{ccc}1 & 0 & 0 \\ 0 & 1 & c_{1} \\ 0 & 0 & 1\end{array}\right\|$ and $B(x)(4)$, where $\begin{array}{lcc}b_{2}(x)=b_{2}^{\prime}(x) \phi_{1}(x), & b_{1}(x) \equiv 0, \quad \text { and } & \left\|r_{i j}(x)\right\|_{1}^{3}= \\ 1 & & 0\end{array}$

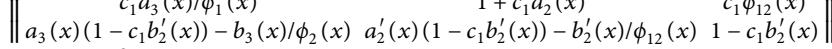
$\left\|r_{i j}(x)\right\|_{1}^{3} \in G L(3, \mathbf{C}[x])$. We make sure that the constructed matrices $\left\|s_{i j}\right\|_{1}^{3}, B(x),\left\|r_{i j}(x)\right\|_{1}^{3}$ together with the original matrix $A(x)$ satisfy equality (5). In order not to enter new notations, we assume that conditions $a_{3}\left(\beta_{1}\right) \neq a_{3}\left(\beta_{2}\right)$ and $a_{2}\left(\beta_{1}\right)=0$ are satisfied for $A(x)$. In the second step in class $\{P F(x) Q(x)\}$ from $A(x)$, we pass to matrix $B(x)$ with the properties specified in proposition. To do this, first, from equation $\left(a_{3}\left(\beta_{2}\right)-a_{3}\left(\beta_{1}\right)\right) c_{2}=a_{2}^{\prime}\left(\beta_{2}\right)$, we find $c_{2} \neq 0$ and then, from congruence, $b_{2}^{\prime}(x)+c_{2}\left(a_{3}(x)-a_{3}\left(\beta_{1}\right)\right)-$ $a_{2}^{\prime}(x) \equiv 0\left(\bmod \phi_{21}(x)\right)$, we find $b_{2}^{\prime}(x)$, $\operatorname{deg} b_{2}^{\prime}(x)<\operatorname{deg} \phi_{12}(x)$. According to the found $c_{2}, b_{2}^{\prime}(x)$, we construct matrices $\left\|s_{i j}\right\|_{1}^{3}=\left\|\begin{array}{ccc}1 & c_{2} & 0 \\ 0 & 1 & 0 \\ 0 & c_{2} & 1\end{array}\right\|$ and $B(x)(4)$, where

$$
\begin{aligned}
& b_{1}(x) \equiv 0, \quad b_{2}(x)=b_{2}^{\prime}(x) \phi_{1}(x), \quad b_{3}(x)=a_{3}(x), \quad \text { and } \\
& \left\|r_{i j}(x)\right\|_{1}^{3}=\left\|\begin{array}{|ccc}
1 & c_{2} \phi_{1}(x) & 0 \\
0 & 1 & 0 \\
0 & a_{2}^{\prime}(x)-c_{2}\left(a_{3}(x)-a_{3}\left(\beta_{1}\right)\right)-b_{2}^{\prime}(x) / \phi_{12}(x) & 1
\end{array}\right\| \epsilon
\end{aligned}
$$

For the original matrix $A(x)$ and constructed $\left\|s_{i j}\right\|_{1}^{3}$, $B(x)$, and $\left\|r_{i j}(x)\right\|_{1}^{3}$, we check the truth of equality (5). Since $b_{2}^{\prime}\left(\beta_{1}\right)=b_{2}^{\prime}\left(\beta_{2}\right)=0$, then $B(x)$ is the required matrix in class $\{P F(x) Q(x)\}$. The proposition is proved.

Proposition 5. If in matrices $A(x)(2)$ and $B(x)$ (4) of class $\{P F(x) Q(x)\}$ the conditions of Proposition 4 are satisfied and, for $\beta_{1}, \beta_{2} \in M_{2}$, we have $a_{3}\left(\beta_{1}\right) \neq a_{3}\left(\beta_{2}\right)$, $b_{3}\left(\beta_{1}\right) \neq b_{3}\left(\beta_{2}\right)$, and $a_{2}\left(\beta_{1}\right)=a_{2}\left(\beta_{2}\right)=b_{2}\left(\beta_{1}\right)=b_{2}\left(\beta_{2}\right)=0$, then the matrix $\left\|s_{i j}\right\|_{1}^{3}$ in (5) is the upper triangular, where $s_{12}=0$. In addition, $\left(a_{2}^{\prime}(x), \phi_{12}(x)\right)=\left(b_{2}^{\prime}(x), \phi_{12}(x)\right)$.

Proof. If we put in (11) $x=\beta_{1}$ and $x=\beta_{2}$, we have $\left\{\begin{array}{l}s_{32}+s_{12} b_{3}\left(\beta_{1}\right)=0, \\ s_{32}+s_{12} b_{3}\left(\beta_{2}\right)=0,\end{array}\right.$ where we immediately get $s_{32}=s_{12}=$ 0 because $b_{3}\left(\beta_{1}\right) \neq b_{3}\left(\beta_{2}\right)$. Then, (11) will take the form

$$
\begin{aligned}
s_{33} a_{2}^{\prime}(x)= & s_{13} a_{2}^{\prime}(x) b_{3}(x)+b_{2}^{\prime}(x)\left(s_{22}+s_{23} a_{2}^{\prime}(x)\right) \\
& +\phi_{12}(x) r_{32}(x) .
\end{aligned}
$$

From (12), it is seen that $\left(a_{2}^{\prime}(x), \phi_{12}(x)\right)=$ $\left(b_{2}^{\prime}(x), \phi_{12}(x)\right)$, since $s_{22}, s_{33} \neq 0$. The proposition is proved.

Denote by $N_{1}$ and $N_{2}$ the subsets of the set $M_{2}$, for elements $\beta_{k} \in N_{1}$ and $\gamma_{l} \in N_{2}$ of which we have $a_{3}\left(\beta_{k}\right) \neq 0$ and $a_{2}\left(\gamma_{l}\right) \neq 0$, respectively. Propositions 2 and 5 imply the following.

Corollary 1. Subsets $N_{1}=\left\{\beta_{k}, \beta_{k} \in M_{2}, a_{3}\left(\beta_{k}\right) \neq 0\right\}$ and $N_{2}=\left\{\gamma_{l}, \gamma_{l} \in M_{2}, a_{2}\left(\gamma_{l}\right) \neq 0\right\}$ for class $\{P F(x) Q(x)\}$ for fixed $\beta_{1}, \beta_{2} \in M_{2}$ do not depend on the choice of the matrix $A(x)$, which satisfies the conditions of Proposition 5.

Since we have imposed the condition that, in (10), $m>1$, then $N_{1}$ is not an empty set. If $N_{2}$ is empty, then $a_{2}(x) \equiv 0$. In this case, the problem of ssk.e. for $3 \times 3$-matrices is reduced to a similar problem for $2 \times 2$-matrices. The latter, as mentioned in Section 1, is resolved in [6]. Therefore, in the future, we assume that $N_{2}$ is not an empty set.

Let $A(x)$ satisfy the conditions of Proposition 5. It is clear that one of the values $a_{3}\left(\beta_{1}\right)$ and $a_{3}\left(\beta_{2}\right)$ is nonzero. Let $a_{3}\left(\beta_{1}\right) \neq 0$. We can also assume that, in $A(x)$, one of the following conditions is fulfilled: $a_{3}\left(\beta_{3}\right)=a_{2}^{\prime}\left(\beta_{3}\right)=1$ for some $\beta_{3} \in N_{1} \cap N_{2}$ if the intersection $N_{1} \cap N_{2}$ is not empty or $a_{3}\left(\beta_{3}\right)=a_{2}^{\prime}\left(\beta_{3}\right)=1$ for some $\beta_{3} \in N_{2}$ otherwise. Each of these conditions for $A(x)$ is achieved by multiplying the first two rows and the first two columns by the corresponding numerical factors. Such a matrix $A(x)$ is called oriented by characteristic roots $\beta_{1}, \beta_{2}, \beta_{3}$ reduced matrix.

Proposition 6. If the intersection $N_{1} \cap N_{2}$ for oriented by the same characteristic roots $\beta_{1}, \beta_{2}, \beta_{3}$ reduced matrices $A(x)$ (2) and $B(x)$ (4) of class $\{P F(x) Q(x)\}$ is not empty, then $s_{11}=$ $s_{22}$ in the matrix $\left\|s_{i j}\right\|_{1}^{3}$ of equation (5).

Proof. According to Proposition 5, the matrix $\left\|s_{i j}\right\|_{1}^{3}$ in question is an upper triangular, and $s_{12}=0$. Equations (9) and (12) hold for elements of matrices $A(x)$ and $B(x)$. From them, we can get a congruence: 


$$
s_{11} a_{2}^{\prime}(x) b_{3}(x)-s_{22} b_{2}^{\prime}(x) a_{3}(x) \equiv 0\left(\bmod \phi_{12}(x)\right)
$$

If we put, in (13), $x=\beta_{3}$, we immediately get $s_{11}-s_{22}=0$. The proposition is proved.

\section{Conditions of Semiscalar Equivalence of Reduced Matrices}

Theorem 1. Let $A(x)$ (2) and $B(x)$ (4) be oriented by the same characteristic roots $\beta_{1}, \beta_{2}, \beta_{3}$ reduced matrices and $\Omega_{A}(x):=\operatorname{det}\left\|\begin{array}{ccc}a_{3}(x) & a_{2}^{\prime}(x) & 1 \\ a_{3}\left(\beta_{1}\right) & 0 & 1 \\ 1 & 1 & 1\end{array}\right\|, \Omega_{B}(x):=\operatorname{det}\left\|\begin{array}{ccc}b_{3}(x) & b_{2}^{\prime}(x) & 1 \\ b_{3}\left(\beta_{1}\right) & 0 & 1 \\ 1 & 1 & 1\end{array}\right\|$.

If, for the sets $N_{1}$ and $N_{2}$ defined in the corollary, the intersection $N_{1} \cap N_{2}$ is not empty, then the matrices $A(x)$ and $B(x)$ are ssk.e. if and only if the following conditions are met:

(i) $a_{2}^{\prime}(\alpha) / a_{3}(\alpha)=b_{2}^{\prime}(\alpha) / b_{3}(\alpha)$ for each $\alpha \in N_{1} \cap N_{2}$.

(ii) Values $\Omega_{A}(\beta), \Omega_{B}(\beta)$ are simultaneously equal to zero or are nonzero for each $\beta \in N_{1} \cup N_{2}$.

(iii) If $\Omega_{A}\left(\beta_{i}\right) \neq 0$ for some $\beta_{i} \in N_{1}$ or $\Omega_{A}\left(\gamma_{j}\right) \neq 0$ for some $\gamma_{j} \in N_{2}$, then

$$
\begin{gathered}
\frac{\Omega_{A}\left(\beta_{k}\right) a_{3}\left(\beta_{i}\right)}{\Omega_{A}\left(\beta_{i}\right) a_{3}\left(\beta_{k}\right)}=\frac{\Omega_{B}\left(\beta_{k}\right) b_{3}\left(\beta_{i}\right)}{\Omega_{B}\left(\beta_{i}\right) b_{3}\left(\beta_{k}\right)}, \\
\frac{\Omega_{A}\left(\gamma_{l}\right) a_{3}\left(\beta_{i}\right)}{\Omega_{A}\left(\beta_{i}\right) a_{2}^{\prime}\left(\gamma_{l}\right)}=\frac{\Omega_{B}\left(\gamma_{l}\right) b_{3}\left(\beta_{i}\right)}{\Omega_{B}\left(\beta_{i}\right) b_{2}^{\prime}\left(\gamma_{l}\right)},
\end{gathered}
$$

or

$$
\begin{gathered}
\frac{\Omega_{A}\left(\beta_{k}\right) a_{2}^{\prime}\left(\gamma_{j}\right)}{\Omega_{A}\left(\gamma_{j}\right) a_{3}\left(\beta_{k}\right)}=\frac{\Omega_{B}\left(\beta_{k}\right) b_{2}^{\prime}\left(\gamma_{j}\right)}{\Omega_{B}\left(\gamma_{j}\right) b_{3}\left(\beta_{k}\right)}, \\
\frac{\Omega_{A}\left(\gamma_{l}\right) a_{2}^{\prime}\left(\gamma_{j}\right)}{\Omega_{A}\left(\gamma_{j}\right) a_{2}^{\prime}\left(\gamma_{l}\right)}=\frac{\Omega_{B}\left(\gamma_{l}\right) b_{2}^{\prime}\left(\gamma_{j}\right)}{\Omega_{B}\left(\gamma_{j}\right) b_{2}^{\prime}\left(\gamma_{l}\right)},
\end{gathered}
$$

for each $\beta_{k} \in N_{1}$ and $\gamma_{l} \in N_{2}$.

If the intersection $N_{1} \cap N_{2}$ is empty, then the matrices $A(x)$ and $B(x)$ are ssk.e. if and only if the following conditions are met:

(1) Values $a_{3}\left(\beta_{k}\right)-1$ and $b_{3}\left(\beta_{k}\right)-1$, as well as values $a_{2}^{\prime}\left(\gamma_{l}\right)-1$ and $b_{2}^{\prime}\left(\gamma_{l}\right)-1$, are simultaneously equal to zero or are nonzero for each $\beta_{k} \in N_{1}$ and $\gamma_{l} \in N_{2}$.

(2) If $a_{3}\left(\beta_{i}\right) \neq 1$ for some $\beta_{i} \in N_{1}$, then

$$
\frac{\left(a_{3}\left(\beta_{k}\right)-1\right) a_{3}\left(\beta_{i}\right)}{\left(a_{3}\left(\beta_{i}\right)-1\right) a_{3}\left(\beta_{k}\right)}=\frac{\left(b_{3}\left(\beta_{k}\right)-1\right) b_{3}\left(\beta_{i}\right)}{\left(b_{3}\left(\beta_{i}\right)-1\right) b_{3}\left(\beta_{k}\right)},
$$

for every $\beta_{k} \in N_{1}$, and if $a_{2}^{\prime}\left(\gamma_{j}\right) \neq 1$ for some $\gamma_{j} \in N_{2}$, then

$$
\frac{\left(a_{2}^{\prime}\left(\gamma_{l}\right)-1\right) a_{2}^{\prime}\left(\gamma_{j}\right)}{\left(a_{2}^{\prime}\left(\gamma_{j}\right)-1\right) a_{2}^{\prime}\left(\gamma_{l}\right)}=\frac{\left(b_{2}^{\prime}\left(\gamma_{l}\right)-1\right) b_{2}^{\prime}\left(\gamma_{j}\right)}{\left(b_{2}^{\prime}\left(\gamma_{j}\right)-1\right) b_{2}^{\prime}\left(\gamma_{l}\right)},
$$

for each $\gamma_{l} \in N_{2}$.

Proof (necessity). Let $A(x) \approx B(x)$. Then, for the elements of matrices $A(x)$ and $B(x)$ are equations (9) and (12), in which according to Proposition 6 in the absence of intersection of sets $N_{1}$ and $N_{2}$ we have $s_{11}=s_{22}$. Therefore, from (13), we immediately have (i). If in equations (9) and (12) put $x=\beta_{1}$, $x=\beta_{3}, x=\beta_{k}$, and $x=\gamma_{l}$, where $\beta_{k}$ and $\gamma_{l}$ mean an arbitrary element of $N_{1}$ and $N_{2}$, respectively, the result can be written in the matrix form as follows:

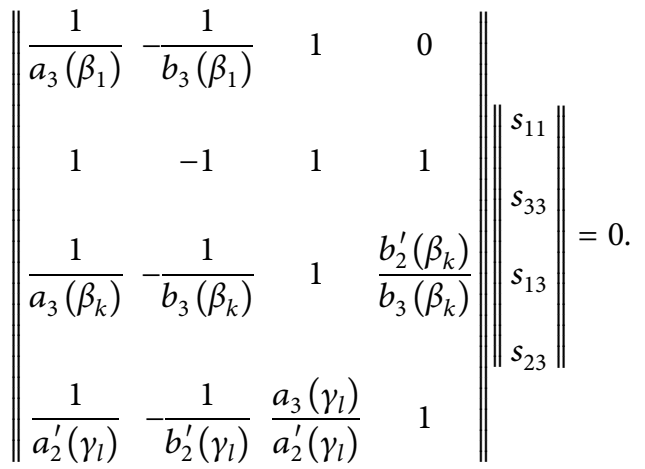

Hence, we have

$$
\left\|\begin{array}{cccc}
\frac{1}{a_{3}\left(\beta_{1}\right)} & -\frac{1}{b_{3}\left(\beta_{1}\right)} & 1 & 0 \\
1-\frac{1}{a_{3}\left(\beta_{1}\right)} & -\left(1-\frac{1}{b_{3}\left(\beta_{1}\right)}\right) & 0 & 1 \\
\frac{\Omega_{A}\left(\beta_{k}\right)}{a_{3}\left(\beta_{k}\right) a_{3}\left(\beta_{1}\right)} & -\frac{\Omega_{B}\left(\beta_{k}\right)}{b_{3}\left(\beta_{k}\right) b_{3}\left(\beta_{1}\right)} & 0 & 0 \\
\frac{\Omega_{A}\left(\gamma_{l}\right)}{a_{2}^{\prime}\left(\gamma_{l}\right) a_{3}\left(\beta_{1}\right)} & -\frac{\Omega_{B}\left(\gamma_{l}\right)}{b_{2}^{\prime}\left(\gamma_{l}\right) b_{3}\left(\beta_{1}\right)} & 0 & 0
\end{array}\right\|\left\|s_{33}\right\|=0 .
$$

From the last equality $(i i)$, since $s_{11}, s_{33} \neq 0$. If $\Omega_{A}\left(\beta_{i}\right) \neq 0$ for some $\beta_{i} \in N_{1}$, then, from equality, $\left\|\begin{array}{ll}\Omega_{A}\left(\beta_{i}\right) / a_{3}\left(\beta_{i}\right) a_{3}\left(\beta_{1}\right) & \Omega_{B}\left(\beta_{i}\right) / b_{3}\left(\beta_{i}\right) b_{3}\left(\beta_{1}\right) \\ \Omega_{A}\left(\beta_{k}\right) / a_{3}\left(\beta_{k}\right) a_{3}\left(\beta_{1}\right) & \Omega_{B}\left(\beta_{k}\right) / b_{3}\left(\beta_{k}\right) b_{3}\left(\beta_{1}\right) \\ \Omega_{A}\left(\gamma_{l}\right) / a_{2}^{\prime}\left(\gamma_{l}\right) a_{3}\left(\beta_{1}\right) & \Omega_{B}\left(\gamma_{l}\right) / b_{2}^{\prime}\left(\gamma_{l}\right) b_{3}\left(\beta_{1}\right)\end{array}\right\|\left\|s_{11}\right\|=0$.

For arbitrary $\beta_{k} \in N_{1}$ and $\gamma_{l} \in N_{2}$, we have each equality with (14), since $s_{11}, s_{33} \neq 0$. If $\Omega_{A}\left(\gamma_{j}\right) \neq 0$ for some $\gamma_{j} \in N_{2}$, then, for any $\beta_{k} \in N_{1}$ and $\gamma_{l} \in N_{2}$, similarly we have each of equation (15).

Now, let the sets $N_{1}, N_{2}$ not intersect and $\beta_{k} \in N_{1}$ and $\gamma_{l} \in N_{2}$. If we put $x=\beta_{1}, x=\beta_{k}$ and $x=\beta_{3}, x=\gamma_{l}$, respectively, in (9) and (12), we obtain equations that can be written as 


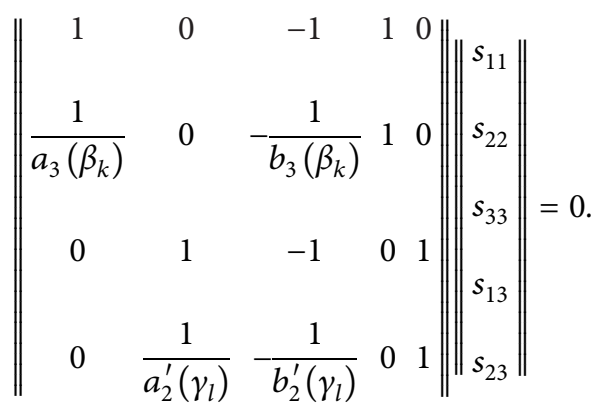

Hence,

we

have

$$
\left\|\begin{array}{ccc}
1-1 / a_{3}\left(\beta_{k}\right) & 0 & -\left(1-1 / b_{3}\left(\beta_{k}\right)\right) \\
0 & 1-1 / a_{2}^{\prime}\left(\gamma_{l}\right) & -\left(1-1 / b_{2}^{\prime}\left(\gamma_{l}\right)\right)
\end{array}\right\|\left\|\begin{array}{l}
s_{11} \\
s_{22} \\
s_{33}
\end{array}\right\|=0 .
$$

Since $s_{11}, s_{22}, s_{33} \neq 0$, then, from the last equality, we obtain $(j)$. If $a_{3}\left(\beta_{i}\right) \neq 1$, for some $\beta_{i} \in N_{1}$, then, from equality,

$$
\left\|\begin{array}{ccc}
1-\frac{1}{a_{3}\left(\beta_{i}\right)} & 0 & -\left(1-\frac{1}{b_{3}\left(\beta_{i}\right)}\right) \\
1-\frac{1}{a_{3}\left(\beta_{k}\right)} & 0 & -\left(1-\frac{1}{b_{3}\left(\beta_{k}\right)}\right)
\end{array}\right\|\|\|_{s_{11}} s_{22} \|=0 .
$$

We get (16) for each $\beta_{k} \in N_{1}$. Similarly, if $a_{2}^{\prime}\left(\gamma_{j}\right) \neq 1$, for some $\gamma_{j} \in N_{2}$, then, from equality,

$$
\left\|\begin{array}{ccc}
0 & 1-\frac{1}{a_{2}^{\prime}\left(\gamma_{j}\right)}-\left(1-\frac{1}{b_{2}^{\prime}\left(\gamma_{j}\right)}\right)\|\| s_{11} \\
s_{22} \\
s_{33}
\end{array}\right\|=0 .
$$

We obtain (17) for every $\gamma_{l} \in N_{2}$. The necessity is proved.

5.1. Sufficiency. Let the sets $N_{1}, N_{2}$ intersect. Consider (19) as an equation with the unknown $\left\|\begin{array}{llll}s_{11} & s_{33} & s_{13} & s_{23}\end{array}\right\|^{T}$. We mean that $\beta_{k}$ and $\gamma_{l}$ acquire all values from sets $N_{1}$ and $N_{2}$, respectively. Therefore, the matrix on the left side of this equation may have more rows than four. Under the conditions of the theorem, the rank of this matrix is equal to 2 if $\Omega_{A}\left(\beta_{k}\right)=\Omega_{A}\left(\gamma_{l}\right)=0$ for every $\beta_{k} \in N_{1}$ and $\gamma_{l} \in N_{2}$. Otherwise, when $\Omega_{A}\left(\beta_{i}\right) \neq 0$ for some $\beta_{i} \in N_{1}$ or $\Omega_{A}\left(\gamma_{j}\right) \neq 0$ for some $\gamma_{j} \in N_{2}$, the rank of the matrix is 3 . In each case, this

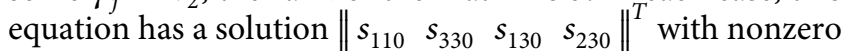
first two components $s_{110}, s_{330}$. If we take into account (i), then, from (19), we can proceed to the equivalent equation (18). Since, as already mentioned, $\beta_{k}$ and $\gamma_{l}$ acquire all values from $N_{1}$ and $N_{2}$, respectively; based on the solution $\left\|\begin{array}{llll}s_{110} & s_{330} & s_{130} & s_{230}\end{array}\right\|^{T}$ of equation (18), we can write such a system of congruences:

$$
\left\{\begin{array}{l}
s_{330} a_{3}(x)-b_{3}(x)\left(s_{110}+s_{130} a_{3}(x)\right)-s_{230} a_{3}(x) b_{2}^{\prime}(x) \equiv 0\left(\bmod \phi_{12}(x)\right), \\
s_{330} a_{2}^{\prime}(x)-b_{2}^{\prime}(x)\left(s_{220}+s_{230} a_{2}^{\prime}(x)\right)-s_{130} a_{2}^{\prime}(x) b_{3}(x) \equiv 0\left(\bmod \phi_{12}(x)\right),
\end{array}\right.
$$

where $s_{220}=s_{110}$ and $s_{110}, s_{330} \neq 0$. The first of the congruences is performed modulo $\phi_{2}(x)=\phi_{1}(x) \phi_{12}(x)$ because $\left(\phi_{1}(x), \phi_{12}(x)\right)=1$ and $\phi_{1}(x)$ divides $a_{3}(x)$ and $b_{3}(x)$. Also, we can pass on module $\phi_{2}(x)$ and in the second congruence after its multiplication by $\phi_{1}(x)$. So, we obtain $\left\{s_{330} a_{3}(x)-b_{3}(x)\left(s_{110}+s_{130} a_{3} \quad(x)\right)-s_{230} a_{3}(x) b_{2}^{\prime}(x) \equiv\right.$ $0\left(\bmod \phi_{2}(x)\right), s_{330} a_{2}(x)-b_{2}(x)\left(s_{220}+s_{230} a_{2}^{\prime}(x)\right)-s_{130} a_{2}$ $(x) b_{3}(x) \equiv 0\left(\bmod \phi_{2}(x)\right)$.

From the elements of solution $\left\|\begin{array}{lllll}s_{110} & s_{330} & s_{130} & s_{230}\end{array}\right\|^{T}$, we construct a matrix $\left\|s_{i j}\right\|_{1}^{3}=\left\|\begin{array}{ccc}s_{110} & 0 & s_{130} \\ 0 & s_{220} & s_{230} \\ 0 & 0 & s_{330}\end{array}\right\|$, where, as before, $s_{220}=s_{110}$, and the matrix $\left\|r_{i j}(x)\right\|_{1}^{3}$, where $r_{11}(x)=$ $s_{110}+s_{130} a_{3}(x), \quad r_{12}(x)=s_{130} a_{2}(x), \quad r_{13}(x)=s_{130} \phi_{2}(x)$, $r_{21}(x)=s_{230} a_{3}(x) / \phi_{1}(x) \in C[x], r_{22}(x)=s_{220}+s_{230} a_{2}^{\prime}(x)$, $r_{23}(x)=s_{230} \phi_{12}(x), \quad r_{31}(x)=\left(s_{330} a_{3}(x)-b_{3}(x) \quad\left(s_{110}+\right.\right.$ $\left.\left.s_{130} a_{3}(x)\right)-s_{230} a_{3}(x) b_{2}^{\prime}(x)\right) / \phi_{2}(x) \in C[x], r_{31}(x)=\left(s_{330} a_{2}\right.$ $\left.(x)-b_{2}(x)\left(s_{220}+s_{230} a_{2}^{\prime}(x)\right)-s_{130} a_{2}(x) b_{3}(x)\right) / \phi_{2}(x) \in C$ $[x]$, and $r_{33}(x)=s_{330}-s_{130} b_{3}(x)-s_{230} b_{2}^{\prime}(x)$.

For the constructed matrices $\left\|s_{i j}\right\|_{1}^{3}$ and $\left\|r_{i j}(x)\right\|_{1}^{3}$ and for the original $A(x)$ and $B(x)$, we are convinced of the truth of equality (5). This means that $A(x) \approx B(x)$.
Now, let set that $N_{1}$ and $N_{2}$ do not intersect. Consider (21) and (22) together as a system of two equations with the unknown $\left\|s_{11} s_{22} s_{33}\right\|^{T}$. Note that, in this system $\beta_{k}$ and $\gamma_{l}$, acquire all values from $N_{1}$ and $N_{2}$, respectively. Under the conditions (1) and (2) of the theorem, the rank of the matrix of this system does not exceed 2. It is also easy to see that this system has a solution of $\left\|s_{110} s_{220} s_{330}\right\|^{T}$ with nonzero of all three of its components, i.e., $s_{110}, s_{220}, s_{330} \neq 0$. If we consider (20) as an equation with the unknown $\left\|\begin{array}{lllll}s_{11} & s_{22} & s_{33} & s_{13} & s_{23}\end{array}\right\|^{T}$ and put in this equation $\left\|\begin{array}{llll}s_{110} & s_{220} & s_{330}\end{array}\right\|^{T}$ instead of $\left\|\begin{array}{lll}s_{11} & s_{22} & s_{33}\end{array}\right\|^{T}$, we can unambiguously find $s_{13}=s_{130}$ and $s_{23}=s_{230}$. This will find the solution $\left\|s_{110} \quad s_{220} s_{330} s_{130} s_{230}\right\|^{T}$ of equation (20) with nonzero of first three components. Based on this solution, we can write a system of congruences (23). Then, by the same considerations as in the previous case, we come to the conclusion that $A(x) \approx B(x)$. The theorem is proved.

\section{Conclusions}

In this paper, a special form is established for the selected set of matrices of simple structure, which is achieved with the help of ssk.e. transformations and is called the oriented by 
characteristic roots reduced matrix (Proposition 4). The matrix of this special shape is lower triangular and has invariant factors on the main diagonal and some predefined properties. Propositions $1-3$ and 5 and Corollary indicate the invariants of the reduced matrix. Propositions 5 and 6 set out the form of the left transform matrix, which together with some right transform (polynomial) matrix translates one reduced matrix into another within class $\{P F(x) Q(x)\}$. This matrix has an upper triangular form with a zero element in position $(1,2)$. In some cases, it has the same first two diagonal elements. The theorem specifies the necessary and sufficient conditions under which the reduced matrices are ssk.e. Thus, Propositions 1-3 and 5, corollary, and theorem indicate a complete system of invariants of the reduced matrix with respect to ssk.e. It is clear that theorem can be applied to establish ssk.e for arbitrary matrices $F(x)$ and $G(x)$ (not reduced) if they belong to the set selected in this paper. Namely, $F(x)$ and $G(x)$ are ssk.e. if and only if their oriented by the same characteristic roots reduced matrices $A(x)$ and $B(x)$ satisfy the conditions of theorem. Also, as already mentioned in Section 1, everything described in this article can be extended to $3 \times 3$-matrices, which after division by the first invariant factor become matrices of simple structure. The proved theorem can be applied to the problem of classification of finite sets of numerical matrices with accuracy to similarity. In particular, let sets $F$ and $G$ of numerical (from the field) $3 \times 3$-matrices be given. On the matrices of these sets, as on the coefficients, we construct matrix polynomials $F(x)$ and $G(x)$ with single higher coefficients. Suppose that, after division by the first invariant factor of the polynomial matrices $F(x)$ and $G(x)$ obtained in this way, we have particles $F^{\prime}(x)$ and $G^{\prime}(x)$ of simple structure. Then, for the similarity of sets $F$ and $G$, it is necessary and sufficient to satisfy the conditions of theorem for consolidated matrices $A(x)$ and $B(x)$ such that $A(x) \approx F^{\prime}(x)$ and $B(x) \approx G^{\prime}(x)$.

\section{Data Availability}

The data used to support the findings of the study are given within the article as references.

\section{Conflicts of Interest}

The author declares that there are no conflicts of interest.

\section{References}

[1] P. S. Kazimirskii, Factorization of Matrix Polynomials, Naukova Dumka, Kyiv, Ukraine, 1981.

[2] P. S. Kazimirskii and V. M. Petrychkovych, "On the equivalence of polynomials matrices," In Theoretical and Applied Problems in Algebra and Differential Equations, pp. 61-66, Naukova Dumka, Kyiv, Ukraine, 1977.

[3] F. R. Gantmacher, The Theory of Matrices, Chelsea Publishing Company, New York, USA, 1959.

[4] P. Lancaster, The Theory of Matrices, Academic Press, New York, USA, 1969.

[5] R. A. Horn and C. R. Johnson, Matrix Analysis, Cambridge University Press, Cambridge, UK, 2012.
[6] B. Z. Shavarovskii, "Toeplitz matrices in the problem of semiscalar equivalence of second-order polynomial matrices," International Journal of Analysis, vol. 2017, Article ID 6701078, 14 pages, 2017.

[7] B. Z. Shavarovskii, "Reduced triangular form of polynomial 3by-3 matrices with one characteristic root and its invariants," Journal of Mathematics, vol. 2018, Article ID 3127984, 6 pages, 2018. 